\title{
Can Pulsed Radiofrequency of the Occipital Nerves Cause Sedation? A New Perspective of Existing Knowledge
}

\author{
Chrysa Arvaniti ${ }^{1,2}$, Alia Ibrahim Madi ${ }^{1}$, Georgia Kostopanagiotou ${ }^{1}$ and Chrysanthi Batistaki ${ }^{1, *}$ \\ ${ }^{1}$ Department of Anaesthesiology, Faculty of Medicine, Attikon University Hospital, National and Kapodistrian University of Athens, Athens, Greece \\ ${ }^{2}$ Department of Neurology, Faculty of Medicine, Attikon University Hospital, National and Kapodistrian University of Athens, Athens, Greece \\ "Corresponding author: Department of Anaesthesiology, Faculty of Medicine, Attikon University Hospital, National and Kapodistrian University of Athens, Athens, Greece. \\ Email: chrysabatistaki@yahoo.gr
}

Received 2019 July 17; Revised 2020 February 23; Accepted 2020 March 19.

\begin{abstract}
Background: Pulsed radiofrequency stimulation (PRF) of the greater and lesser occipital nerves (GON and LON) has neuromodulative and analgesic properties.

Objectives: The aim of this study was to investigate whether the PRF of the occipital nerves can cause sedation.

Methods: This is an observational case series study in Pain Management Unit of Attikon University Hospital. Patients suffering from primary headaches were scheduled for PRF of occipital nerves following a standardized protocol. The Bispectral Index device was applied and recorded every minute throughout the procedure.

Results: 22 patients were studied. BIS values were lowered in all but three patients during GON stimulation, and in all patients during LON stimulation. Values of decline compared to baseline ranged between 0 - 23 (median 8.5) for GON and 1 - 27 (median 14 ) for LON, with LON decline being significantly lower.

Conclusions: PRF at the occipital nerves led to mild sedation in all patients. Further studies are required to investigate this effect and clarify the exact mode of action of pulsed radiofrequency.
\end{abstract}

Keywords: Headache, Pulsed Radiofrequency, Sedation

\section{Background}

Pulsed radiofrequency stimulation (PRF) of the occipital nerves (greater and lesser) is a minimally invasive technique applied in patients suffering from various types of headaches $(1,2)$. It is currently indicated for chronic migraine, tension and cluster headache, as well as in cases of occipital neuralgia $(1,2)$, following specific criteria and a positive diagnostic blockade of the occipital nerves with a local anesthetic (1-8).

Pulsed radiofrequency has gained significant scientific interest during the last decade due to its neuromodulative properties, and its non-destructive nature (9). It is believed that it results in alteration of the synaptic transmission of the sensory nerves in addition to a neuromodulatorytype effect (1). The mode of action of PRF is through the application of an electric field of low intensity around the sensory nerves (in this case the occipital nerves), an action which leads to decreased conduction through the pain fibers, mostly of type $\mathrm{C}$, while not affecting the myelinated ones $(1,2)$. Many studies also suggest that PRF may increase the inhibitory action of serotonergic, noradrener- gic, and endogenous opioid pathways $(1,2,10)$, contributing to pain relief. However, enhancement of these pathways may also affect other functions, causing mild sedation, or even stress reduction.

\section{Objectives}

This observation is to be tested in the current study, which aims to investigate the effect of pulsed radiofrequency stimulation via a standardized protocol to the occipital nerves (greater and lesser) on the level of sedation, as measured by the Bispectral Index device.

\section{Methods}

All patients enrolled were patients who were followed up at the Pain Management Unit of "Attikon" University Hospital, after written informed consent. Patients were scheduled for therapy, following the standard diagnostic and therapeutic pain protocol, consisting of thorough 
neurological examination, systemic pharmacotherapy, diagnostic occipital nerve blocks, and then pulsed radiofrequency stimulation of the occipital nerves.

Participating patients suffered from primary headaches, mostly migraine, as well as cluster headache and occipital neuralgia. They have all in the past received systemic pharmacotherapy for their headache (prophylaxis with topiramate or amitriptylline and tryptanes as rescue treatment), and they have all responded positively (with $>50 \%$ pain relief after occipital nerve block with a local anesthetic). All patients included in the study did not have an adequate response to systemic pharmacotherapy, pain over the crisis of $>4 / 10$, occipital tenderness bilaterally or unilaterally, and days with headache of more than 10/month. Exclusion criteria were age $<18$ years old, pregnancy or lactation, presence of a cardiac pacemaker, previous therapy with PRF, severe psychiatric disorder, and patient refusal.

All patients were managed using the same PRF devices [Diros RF hybrid cannula (integrated probe), 22G, $54 \mathrm{~mm}$, $4 \mathrm{~mm}$ active tip, Diros Owl quodropolar generator]. After an intravenous line, basic monitoring (ECG, BP, and SPO2) and Bispectral Index placement (Medronic/Covidien, USA) being applied at the patient's forehead, the procedure was performed using aseptic conditions, without local anesthesia in order to identify and correctly stimulate the nerve. At first, the two greater occipital nerves (GON) were stimulated ( 40 - $60 \mathrm{~V}, 2 \mathrm{~Hz}$, impedance 150 - $400 \Omega$, plateau temperature $42^{\circ} \mathrm{C}$, time: 6 minutes), followed by the lesser (LON) ( 40 - $60 \mathrm{~V}, 2 \mathrm{~Hz}$, impedance 150 - $400 \Omega$, plateau temperature $42^{\circ} \mathrm{C}$, time: 6 minutes). Nerves were identified following the anatomical landmarks described in the literature (4), with the target point for the GON being onequarter to one-third of the distance of the line connecting the external occipital protuberance with the mastoid process, medial to the occipital artery. Similarly, for the LON, the target point was located two-thirds of the distance from the occipital protuberance up to the mastoid process. The accurate position of the needle was confirmed using electrical stimulation, with repeated adjustments in order to maximize nerve stimulation at the lower possible voltage (with target being $<0.4 \mathrm{mV}$ ). The technique used was standardized by the Pain Unit team and performed for all patients receiving the same treatment. During the procedure, the Bispectral Index (BIS) indication was recorded every minute until the end of the procedure by an independent researcher, not belonging to the team of the Pain Unit. The BIS device collects the raw EEG data that indicate brain activity in real time. The system uses its clinically validated algorithm to filter, analyze, and correlate the data and the final results are continually calculated and displayed as the $\mathrm{BIS}^{\mathrm{TM}}$ index (a number between 0 and 100), indicating the patient's level of sedation (with 0 indicating complete sedation-coma and 100 indicating complete alertness).

Postoperatively, patients were allowed to recover and then were discharged with written orders. Statistical analysis of results was performed using SPSS V. 15.0, using ANOVA analysis of variance, with P set as $<0.05$.

\section{Results}

Demographic characteristics of patients, type of headache, and baseline BIS values are presented in Table 1. Patients' age ranged between 27 - 69 years old (median 53), and suffered mostly from migraine. Baseline BIS values ranged between 96 and 98 (median 99). The BIS values declined in the majority of patients during GON PRF and in all patients during LON. Values of decline compared to baseline ranged between $0-23$ (median 8.5) for the GON (except for three patients where no reduction was noticed) and between 1-27 (median 14) for the LON, where the decline was noticed in all patients. A statistically significant difference was observed between the decline in BIS units between the greater and the lesser occipital nerves stimulation, with the lesser showing a more significant reduction (Table 1 and Figure 1). The BIS values returned to normal shortly ( $<10$ minutes) after the intervention. No adverse effects were recorded throughout the procedure.

Table 1. Demographic Characteristics, Type of Headache, Bispectral Index (BIS) Values (Baseline and Mean Change) During Pulsed Radiofrequency of the Greater Occipital Nerves (GON), and Lesser Occipital Nerves (LON) in Patients Studied ( $\mathrm{N}=22$ )

\begin{tabular}{|lc|}
\hline Parameters & Values \\
\hline Gender (male/female) & $8 / 14$ \\
\hline Age & $50.04(13.44)$ \\
\hline Weight $(\mathbf{k g})$ & $70.80(12.52)$ \\
\hline Height $(\mathbf{c m})$ & $166.80(7.86)$ \\
\hline Type of headache & \\
\hline \multicolumn{1}{|c|}{ Migraine } & $14(63.63 \%)$ \\
\hline \multicolumn{1}{|c|}{ Cluster } & $5(22.72 \%)$ \\
\hline Occipital neuralgia & $3(13.63 \%)$ \\
\hline Baseline BIS & $97.77(0.52)$ \\
\hline Mean decline in BIS units & \\
\hline GON & $9.09(6.14)$ \\
\hline LON & $12.57(7.63)$ \\
\hline P value & $0.005^{*}$ \\
\hline
\end{tabular}

\section{Discussion}

The concept of pulsed radiofrequency (PRF) has emerged during the last decade, with various targets and 


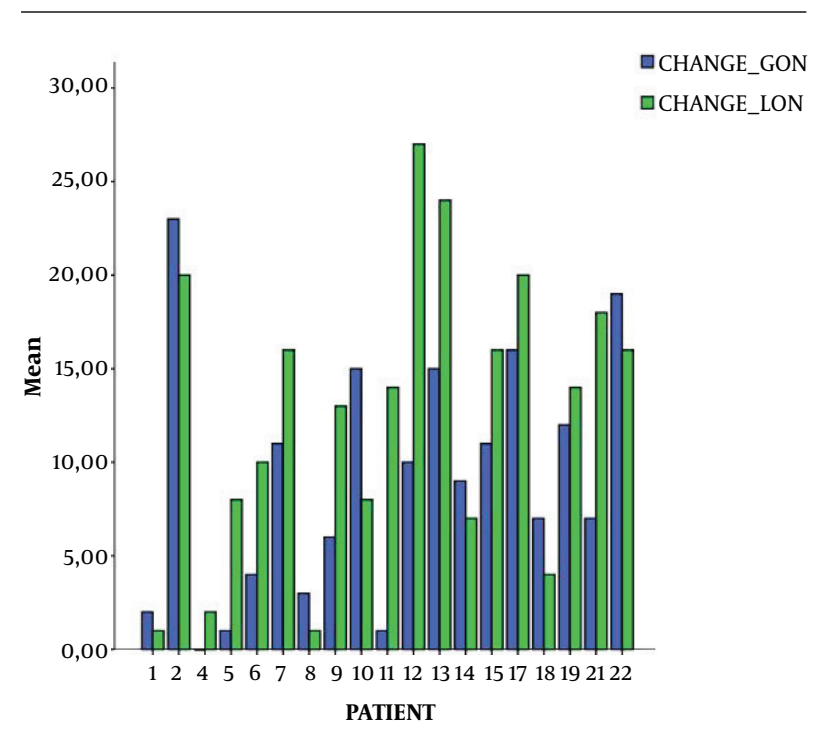

Figure 1. Mean change in Bispectral Index values in greater occipital nerves (GON) and lesser occipital nerves (LON) during pulsed radiofrequency stimulation in each patient $(\mathrm{n}=22)$

efficacy $(2,3)$, depending on the nerve, the type of pain, duration of pain, the patient characteristics, and more. The theory behind PRF is based on the energy created around the tip of the electrode. The tip delivers a large electrical current density, producing heat, estimated to be around $2 \times 10^{4} \mathrm{~A} / \mathrm{m}^{2}(1-3)$. The degree of the heat created at the tip of the insulated needle is directly proportional to the current passing through it and can be variable. In the pulsed form of radiofrequency, the heat created varies between $42^{\circ} \mathrm{C}$ and $44^{\circ} \mathrm{C}$ and produces reversible injury to the target tissue (in this case around the occipital nerves) (1-4). The biological changes caused by pulsed radiofrequency are complex, including a thermal effect, as well as the energy created by the electric field (1). The delivered current $(20 \mathrm{~ms})$ is ultra-short and applied in high voltage bursts. Therefore the phase where no field is provided (the so-called "silent phase", $480 \mathrm{~ms}$ ) allows enough time for tissues to eliminate thermal energy and keep their temperature below $42^{\circ} \mathrm{C}(1,2)$.

The main finding of this study was that in all but three patients, Bispectral Index values decreased, in both greater and lesser occipital nerves, with a significantly greater decline at the lesser. In some cases, the decline reached a number of more than 20 units below baseline, a fact that is considered significant and requires explanation and further investigation.

The precise mode of action of PRF is still not clarified. It has been proposed that this electrical field affects neurons by changing and modulating synaptic signaling, es- pecially via the C-pain fibers, leaving myelinated fibers intact $(7,10)$. Additionally, another theory suggests that PRF alters the transmission of pain signals through modulation of c-Fos, the immediate early gene (7, 11-14), which seems to be independent of the temperature applied at the nerve tissue. The formation and expression of the cFos gene in lamina I and II, is of great importance, since it means that an expansion of the electrical field into the central nervous system is occurring during PRF, resulting in a neuromodulative effect $(1,2,11-17)$. The formation of this gene also leads to the development of the second messenger RNA, a substance called "preprodinorphin", which belongs to the endogenous opioid system, and enhances endorphin production $(1,2,11)$. This finding is in accordance with the work by Hagiwara et al. (18), who investigated the action of pulsed radiofrequency in rats, proving that its antinociceptive properties are also mediated via enhancement of serotonergic, noradrenergic and endogenous opioid inhibitory pain pathways. This evidence, which supports the action of pulsed radiofrequency on the dorsal horn and on the inhibitory pain pathways, may explain the action of the technique on the occipital nerves. It may be that the application of the pulses might induce similar changes at the brain stem, leading to a neuromodulating effect that is immediately seen as a mild sedation, as measured by the bispectral Index. The duration of this effect seemed to be short since, in all patients, BIS returned to baseline values soon after the termination of the procedure, but it is not known how long this effect lasts at a microscopic level.

Another issue that requires further investigation is the fact that radiofrequency application at the lesser occipital nerves resulted in more sedation than the major occipital nerves. However, it is not known whether it is the lesser occipital nerves themselves that anatomically behave differently or the fact that stimulation of the lesser occipital nerves occurred after, the greater ones, leading to a possible additive effect of pulsed radiofrequency, and therefore more sedation. Is it serotonine, endorphines, or both that induce this mild sedation? Indeed, more studies are required to further investigate this effect, and possibly relate it to the exact mode of action of pulsed radiofrequency.

\subsection{Limitations}

A limitation of this study is the small number of patients studied, the absence of randomization, and, of course the absence of another group where the LON are being stimulated before the GON in order to study the possible additive effect. As for BIS and RF generator possible interference, manufacturers of the RF generator, report no such effect, indicating that BIS measurement was accurate during the procedure. 


\subsection{Conclusions}

In conclusion, the application of pulsed radiofrequency at the occipital nerves (greater and lesser) led to mild sedation in all patients, as measured by the Bispectral Index. Further studies are needed in order to investigate this effect and possibly clarify the exact mode of action of pulsed radiofrequency.

\section{Footnotes}

Authors' Contribution: Chrysa Arvaniti, Alia Ibrahim Madi, Georgia Kostopanagiotou, and Chrysanthi Batistaki have equally contributed to the study design and the manuscript intellectual content. Chrysa Arvaniti, Alia Ibrahim Madi, and Chrysanthi Batistaki have equally contributed to the acquisition, analysis, and interpretation of data. All authors have read and approved the final manuscript submitted.

Conflict of Interests: There are absolutely no conflicts of interest regarding this manuscript.

Ethical Approval: http://www.attikonhospital.gr/, 896/29-01-2019.

Funding/Support: There is no funding support for the current study.

Patient Consent: Written informed consent was obtained from all patients.

\section{References}

1. Chua NH, Vissers KC, Sluijter ME. Pulsed radiofrequency treatment in interventional pain management: Mechanisms and potential indications-a review. Acta Neurochir(Wien). 2011;153(4):763-71. doi: 10.1007/s00701-010-0881-5. [PubMed: 21116663]. [PubMed Central: PMC3059755]

2. Bogduk N. Pulsed radiofrequency. Pain Med. 2006;7(5):396-407. doi: 10.1111/j.1526-4637.2006.00210.x. [PubMed: 17014598].

3. Van Zundert J, Patijn J, Hartrick C, Lataster A, Huygen F, Mekhail N, et al. Evidence-based interventional pain medicine according to clinical diagnoses. John Wiley \& Sons, Ltd; 2012.

4. Narouze SN. Interventional management of head and face pain. New York: Springer Science \& Business Media; 2014. doi:10.1007/978-1-46148951-1.
5. Vanneste T, Van Lantschoot A, Van Boxem K, Van Zundert J. Pulsed radiofrequency in chronic pain. Curr Opin Anaesthesiol. 2017;30(5):57782. doi: 10.1097/ACO.0000000000000502. [PubMed: 28700369].

6. Gooriah R, Nimeri R, Ahmed F. Evidence-based treatments for adults with migraine. Pain Res Treat. 2015;2015:629382. doi: 10.1155/2015/629382. [PubMed: 26839703]. [PubMed Central: PMC4709728].

7. Byrd D, Mackey S. Pulsed radiofrequency for chronic pain. Curr Pain Headache Rep. 2008;12(1):37-41. doi: 10.1007/s11916-008-0008-3. [PubMed: 18417022]. [PubMed Central: PMC2913603].

8. Tepper SJ. Advanced interventions for headache. Headache 2012;52 Suppl 1:50-4. doi: 10.1111/j.1526-4610.2012.02133.x. [PubMed: 22540208].

9. Abejon D, Reig E. Is pulsed radiofrequency a neuromodulation technique? Neuromodulation. 2003;6(1):1-3. doi: 10.1046/j.1525 1403.2003.03009.x. [PubMed: 22150906].

10. Cosman ER Jr, Cosman Er Sr. Electric and thermal field effects in tissue around radiofrequency electrodes. Pain Med. 2005;6(6):405-24. doi: 10.1111/j.1526-4637.2005.00076.x. [PubMed: 16336478].

11. Van Zundert J, de Louw AJ, Joosten EA, Kessels AG, Honig W, Dederen PJ, et al. Pulsed and continuous radiofrequency current adjacent to the cervical dorsal root ganglion of the rat induces late cellular activity in the dorsal horn. Anesthesiology. 2005;102(1):125-31. doi: 10.1097/00000542-200501000-00021. [PubMed: 15618796].

12. Hunter JC, Woodburn VL, Durieux C, Pettersson EKE, Poat JA, Hughes J. C-fos antisense oligodeoxynucleotide increases formalin-induced nociception and regulates preprodynorphin expression. Neuroscience. 1995;65(2):485-92. doi: 10.1016/0306-4522(94)00500-5.

13. Higuchi Y, Nashold BS Jr, Sluijter M, Cosman E, Pearlstein RD. Exposure of the dorsal root ganglion in rats to pulsed radiofrequency currents activates dorsal horn lamina I and II neurons. Neurosurgery. 2002;50(4):850-5. discussion 856. doi:10.1097/00006123-200204000 00030. [PubMed: 11904038]

14. Sandkuhler J, Treier AC, Liu XG, Ohnimus M. The massive expression of c-fos protein in spinal dorsal horn neurons is not followed by long term changes in spinal nociception. Neuroscience. 1996;73(3):657-66. doi: 10.1016/0306-4522(96)00073-5. [PubMed: 8809787]

15. Sluijter ME, Imani F. Evolution and mode of action of pulsed radiofrequency. Anesth Pain Med. 2013;2(4):139-41. doi: 10.5812/aapm.10213. [PubMed: 24223349]. [PubMed Central: PMC3821144].

16. Imani F, Gharaei H, Rezvani M. Pulsed radiofrequency of lumbar dorsal root ganglion for chronic postamputation phantom pain. Anesth Pain Med.2012;1(3):194-7. doi: 10.5812/kowsar.22287523.3768. [PubMed: 24904793]. [PubMed Central: PMC4018701].

17. Imani F. Using pulsed radiofrequency for chronic pain. Anesth Pain Med. 2012;1(3):155-6. doi: 10.5812/kowsar.22287523.4047. [PubMed: 24904784]. [PubMed Central: PMC4018683].

18. Hagiwara S, Iwasaka H, Takeshima N, Noguchi T. Mechanisms of analgesic action of pulsed radiofrequency on adjuvant-induced pain in the rat: Roles of descending adrenergic and serotonergic systems. Eur J Pain. 2009;13(3):249-52. doi: 10.1016/j.ejpain.2008.04.013. [PubMed: 18539061]. 